\title{
Clinical Outcome of Spinal Epidural Abscess in Elderly Patients
}

\author{
Jae Hyun Kim${ }^{1}$, Eun Hye Lee ${ }^{3}$, Jiha Kim ${ }^{1,2}$, Choonghyo Kim ${ }^{1,2}$ \\ ${ }^{1}$ Department of Neurosurgery, Kangwon National University Hospital, Chuncheon, \\ 2Department of Neurosurgery, Kangwon National University School of Medicine, Chuncheon, \\ ${ }^{3}$ Department of Neurosurgery, Uijeongbu St. Mary's Hospital, Uijeongbu, Republic of Korea
}

Corresponding author: Choonghyo Kim Department of Neurosurgery, Kangwon National University Hospital, Kangwon National University School of Medicine, 156, Baengnyeong-ro, Chuncheon 24289, Republic of Korea

Tel: +82-33-258-2410

Fax: +82-33-258-9489

E-mail: jeuelkim@gmail.com

Received: August 13, 2020

Revised: August 28, 2020

Accepted: September 7, 2020
Objective: Spinal epidural abscess (SEA) is a rare infection with a high rate of mortality and morbidity, which requires urgent surgery. Although surgery is the mainstay of treatment, some investigators currently report good outcomes after medical treatment. Thus, the optimal treatment option is debatable without established guidelines for standard of care. Moreover, surgery in elderly SEA patients is challenging because of comorbidities and perioperative risks. Methods: We retrospectively reviewed the medical records of 16 elderly patients with SEA, including 6 males and 10 females, ranging in age from 69 to 88 years (mean 75 years). Fourteen patients underwent open surgery, and 2 underwent aspiration biopsy as well as antibiotic treatment. We statistically analyzed variables related to clinical outcomes. The mean follow-up duration was 21.6 months. Results: Twelve patients had comorbidities, and 5 had other systemic infections. Four and 3 patients had a history of spinal intervention and spinal fusion surgery, respectively. Finally, 7 patients completely improved and 2 were moderately disabled. Another 2 patients were severely disabled and 5 died during antibiotic treatment. Most mortalities were due to comorbidities. SEA in elderly patients with comorbidities was associated with high mortality rates. Furthermore, delayed surgery was significantly related to poor neurological outcomes, whereas other variables were not significantly related to the outcomes of SEA in elderly patients. Conclusion: We identified a statistically significant relationship between neurological outcome and comorbidity $(p=$ $0.026)$, predisposing conditions $(p=0.010)$, and time to surgery $(p=0.014)$, suggesting that early surgery should be considered, even in elderly SEA patients.

Key Words: Aged; Epidural abscess; Surgical procedures

\section{INTRODUCTION}

Spinal epidural abscess (SEA) is a rare but potentially devastating infection that requires urgent/emergent surgical treatment ${ }^{6,17,18)}$. Although it was seen in 0.2 to 1.2 cases per 10,000 hospital admissions in the mid-1970s, current estimates of incidence range from 2 to 12.5 per 10,000 admissions ${ }^{6,12,25)}$. This increase likely primarily results from the increased aging population, increased spinal procedures, and intravenous (IV) drug abuse $3,6,15,20,21,26,28)$.

The many advances in antibiotics, more rapid diagnosis by sophisticated imaging, and heightened physician awareness have led to attenuated morbidity and mortality in SEA patients ${ }^{1320)}$. However, SEA continues to have a high mortality rate, ranging from $15 \%$ to $23 \%{ }^{1)}$, and 4 to $22 \%$ of patients suffer irreversible paralysis $^{12)}$.
Although urgent/emergent surgical decompression and IV antibiotic therapy are the cornerstone therapies for SEA, ideal management of this condition remains controversial ${ }^{4,25)}$. Currently, some investigators report favorable outcomes after nonsurgical treatment in select patients ${ }^{2,5,6,14)}$. Thus, optimal treatment for SEA is still debatable without any established guidelines.

Treatment for SEA in elderly patients is more challenging due to the presence of comorbidities and perioperative risks. Many surgeons face a dilemma in the decision to proceed with surgery or medical treatment for these patients. Considering the perioperative risk and/or poor general conditions, medical treatment is often an acceptable option for elderly patients. However, there is a paucity of information concerning the optimal treatment for SEA in elderly patients. Therefore, it is worth conducting a study to analyze the clinical outcomes of SEA in elderly patients. 


\section{MATERIALS AND METHODS}

\section{Patients}

We conducted a retrospective review of 16 patients with SEA older than 65 years who underwent treatment at the department of neurosurgery between January 2006 and December 2014 in a single teaching hospital. A diagnosis of SEA was made clinically and radiologically using a magnetic resonance image (MRI). We analyzed patient medical records, laboratory results, radiological findings, and surgical reports.

Initial neurological status was graded using Bremer's grading system $^{7)}$ for the progression of signs and symptoms. The Charlson comorbid index scoring system" was used to describe the patients' comorbid status. The location of the SEA was identified by the MRI findings and was classified as dorsal or ventral. The size of the SEA was measured according to the number of the adjacent vertebral body. The severity of thecal sac compression was defined as mild (below $1 / 4$ of total area of spinal canal), moderate (below half), or severe (above half) at the maximal compression level of MRI.

Delay in presentation was defined as the time interval between the onset of clinical symptoms and presentation to medical services. Time to surgery was defined as the time interval from admission day to operative day. The neurological outcomes were classified as no symptoms to slightly disabled, moderate to severely disabled (according to modified Rankin grade) and death. The relationship between such variables and neurological outcome was statistically analyzed. We analyzed categorical data with Fisher's exact test and Kruskal-Wallice test with post hoc analysis. We also used linear by linear association test for the verification of trend. Results with $\mathrm{p}$ less than 0.05 were considered significant. Statistical software IBM SPSS Statistics for Windows, version 24.0 (IBM Corp., Armonk, NY, USA) was used for the analysis. The mean follow-up duration was 21.6 months.

\section{Treatment Protocol}

A microbiological study was performed for both peripheral blood and abscess acquired from surgical specimens before initiation of the empirical antibiotic treatment in general ${ }^{12)}$. Then, initial empirical antibiotics were modified according to the final microbiological reports of drug sensitivity for identified pathogens. The choice, initiation and termination of antibiotics were informed by the infectious disease physician. Decisions to perform surgery were made by a well-coordinated team for spinal infection (2 neurosurgeons and one infectious disease physician). We routinely performed urgent surgery in patients with either neurological deficits, high risk of medical treatment failure, or compression of neural tissue due to the huge burden of infection.

We performed open surgery for 14 patients, whereas computed tomography-guided aspiration was performed for 2 patients who could not tolerate open surgery. Two patients, whose causative pathogens were not confirmed microbiologically, underwent empirical antibiotic treatment. For another 2 patients who had delayed surgery, empirical antibiotic treatment was initiated after obtaining peripheral blood for culture study. The patients were typically treated with IV antibiotics for 6 weeks or until symptoms and signs of infection subsided and C-reactive protein (CRP) levels normalized. In case of adverse effects, we modified antibiotics according to recommendations of the infectious disease physician.

\section{RESULTS}

\section{Basic Characteristics}

Sixteen patients were included in the current study, and the male : female ratio was 6:10. Their ages ranged from 69 to 88 years (mean 75 years). Ten out of 16 patients presented with axial pain and/or sciatica. Another 5 exhibited motor weakness, including 3 patients with paraplegia. One presented with an altered mentality resulting from metabolic alkalosis. Delay in presentation ranged from 2 to 90 days (mean 23.9 days). Patient baseline characteristics are summarized in Table 1, and initial clinical manifestations and final neurologic status are summarized in Table 2.

Leukocyte counts varied from 6,500 to 12,480 cells $/ \mathrm{mm}^{3}$. The erythrocyte sedimentation rate and CRP varied from 6 to $120 \mathrm{~mm} / \mathrm{hr}$ and from 32 to $230 \mathrm{mg} / \mathrm{L}$, respectively.

Twelve of 16 patients exhibited one or more comorbidities, and 5 patients had 3 or more comorbidities. Two patients had no comorbid conditions and another 2 patients had only hypertension. Detailed data for comorbidities and associated Charlson comorbidity index scores are summarized in Figure 1 and Table 3, respectively.

\section{Radiological Findings}

All cases involved the lumbar and/or thoracic spine, and none had cervical spine involvement. The size of the SEA was one or 2 vertebral body lengths in most patients (11 out of 16). However, 4 patients had involvement of more than 3 vertebral body lengths. Thirteen patients presented with ventral SEA, and 2 patients exhibited dorsal SEA. One patient had both dorsal and ventral involvement. MRI showed mild, moderate, and severe compression of the dural sac in 7, 4, and 5 patients, respectively. Six patients presented with psoas abscess and 22 patients exhibited vertebral osteomyelitis radiologically on MRI (Table 1). 
Table 1. Basal characteristics of the patients

\begin{tabular}{|c|c|c|c|c|c|c|c|c|c|c|c|c|}
\hline No & $\begin{array}{l}\text { Sex/ } \\
\text { Age }\end{array}$ & $\begin{array}{l}\text { Chief } \\
\text { complaint }\end{array}$ & Fever & $\begin{array}{c}\text { Delay in } \\
\text { presentation (days) }\end{array}$ & $\begin{array}{l}\text { Combined } \\
\text { infection }\end{array}$ & $\begin{array}{l}\text { Size of } \\
\text { abscess }\end{array}$ & $\begin{array}{l}\text { Psoas } \\
\text { abscess }\end{array}$ & Osteomylitis & $\begin{array}{l}\text { Location } \\
\text { of abscess }\end{array}$ & $\begin{array}{l}\text { Thecal sac } \\
\text { compression }\end{array}$ & $\begin{array}{l}\text { Mode of } \\
\text { surgery }\end{array}$ & $\begin{array}{l}\text { Cause of } \\
\text { mortality }\end{array}$ \\
\hline 1 & M69 & Paraplegia & + & 10 & & 7 & + & + & Ventral & Mild & Laminectomy & \\
\hline 2 & $\mathrm{~F} / 71$ & Paraparesis & + & 7 & Sepsis & 2 & + & + & Ventral & Severe & $\begin{array}{c}\text { Anterior }+ \\
\text { Posterior }^{+}\end{array}$ & $\begin{array}{l}\text { Aortic pseu- } \\
\text { doaneurysm }\end{array}$ \\
\hline 3 & N69 & Paraparesis & & 7 & $\begin{array}{l}\text { Cerebellar } \\
\text { abscess }\end{array}$ & 2 & & & Dorsal & Severe & Laminectomy & \\
\hline 4 & $\mathrm{~F} / 75$ & Back pain & & 30 & & 3 & + & + & Both & Moderate & $\begin{array}{c}\text { Anterior }+ \\
\text { Posterior }^{+}\end{array}$ & \\
\hline 5 & $\mathrm{~F} / 84$ & Paraplegia & & 2 & & 4 & & + & Ventral & Mild & Laminectomy & $\begin{array}{l}\text { Cerebral } \\
\text { infarction }\end{array}$ \\
\hline 6 & M69 & Back pain & & 30 & & 2 & + & + & Ventral & Severe & Laminectomy & \\
\hline 7 & $F / 88$ & Back pain & & 14 & & 2 & & + & Ventral & Mild & Aspiration & \\
\hline 8 & M72 & Back pain & & 30 & & 2 & & + & Ventral & Mild & Laminectomy & \\
\hline 9 & $\mathrm{~F} / 77$ & Paraplegia & + & 24 & Pneumonia & 2 & & + & Ventral & Severe & $\begin{array}{c}\text { Anterior }+ \\
\text { Posterior }^{+}\end{array}$ & ARDS \\
\hline 10 & $\mathrm{~F} / 75$ & Back pain & & 7 & & 4 & + & + & Ventral & Moderate & Laminectomy & AMl \\
\hline 11 & $\mathrm{~F} / 74$ & Back pain & & 90 & & 1 & + & + & Ventral & Severe & Laminectomy & \\
\hline 12 & $\mathrm{~F} / 70$ & Back pain & & 10 & & 2 & & + & Dorsal & Mild & FB removal & \\
\hline 13 & $\mathrm{~F} / 74$ & Back pain & & 30 & & 2 & & + & Ventral & Mild & FB removal & \\
\hline 14 & M86 & $\begin{array}{l}\text { Altered } \\
\text { mentality }\end{array}$ & + & 21 & $\begin{array}{l}\text { Pulmonary } \\
\text { abscess }\end{array}$ & 2 & & + & Ventral & Moderate & Aspiration & \\
\hline 15 & M73 & Back pain & & 40 & & 4 & & + & Ventral & Mild & FB removal & \\
\hline 16 & $F / 76$ & Back pain & + & 30 & $\begin{array}{l}\text { Pulmonary } \\
\text { tuberculosis }\end{array}$ & 2 & & + & Ventral & Moderate & Laminectomy & AMl \\
\hline
\end{tabular}

M: male; F: female; FB: foreign body (spinal fusion instruments); ARDS: acute respiratory distress syndrome; AMl: acute myocardial infarction.

The size of spinal epidural abscess was measured according to the number of adjacent vertebral body. ${ }^{+}$Combined surgery via anterior and posterior approach.

Table 2. Clinical manifestation vs. final neurologic status of patients

\begin{tabular}{ccccc}
\hline \hline \multirow{2}{*}{ Staging system for SEA } & \multicolumn{3}{c}{ Final neurological status ${ }^{+}$} & Total number of patients \\
\cline { 2 - 5 } & $0-2$ & $3-4$ & $3-6$ & 8 \\
2 & 3 & 2 & 2 & 3 \\
3 & 3 & & 2 & 3 \\
4 & 1 & & 3 \\
\hline
\end{tabular}

The statistical significance was defined as $p=0.342$ (Fisher's exact test).

SEA: spinal epidural abscess.

'Staging system for SEA is from the Bremer's grading system (2004). ${ }^{\dagger}$ Final neurological status 1 to 5 is from modified Rankin grade and 6 is death.

Table 3. Comorbidity vs. final neurologic status patients

\begin{tabular}{ccccc}
\hline \hline \multirow{2}{*}{ Charlson comorbidity index } & \multicolumn{3}{c}{ Final neurological status ${ }^{*}$ Total number of patients } \\
\cline { 2 - 5 } & $0-2$ & $3-4$ & $5-6$ & 4 \\
1 & 2 & 1 & 1 & 5 \\
2 & 4 & 1 & 2 & 3 \\
3 & 1 & 3 & 3 \\
4 & & & 1 & 3 \\
\hline
\end{tabular}

The statistical significance was defined as $p=0.026$ (linear-to-linear association test).

*Final neurological status 1 to 5 is from modified Rankin grade and 6 is death.

44 www.thenerve.net 
Table 4. Predisposing conditions vs. final neurologic status of patients

\begin{tabular}{lcccc}
\hline \hline \multirow{2}{*}{ Predisposing conditions } & \multicolumn{3}{c}{ Final neurological status } & \multirow{2}{*}{ Total number of patients } \\
\cline { 2 - 4 } & $0-2$ & $3-4$ & $5-6$ & 5 \\
Systemic infection & 5 & 2 & 5 & 7 \\
Spinal intervention & & & 2 & 4 \\
Others & 2 & & 2
\end{tabular}

The statistical significance was defined as $p=0.010$ (Kruskal-Wallis test with post-hoc analysis [between systemic infection and spinal intervention]).

"Final neurological status 1 to 5 is from modified Rankin grade and 6 is death. ${ }^{+}$Spinal operation and epidural block.

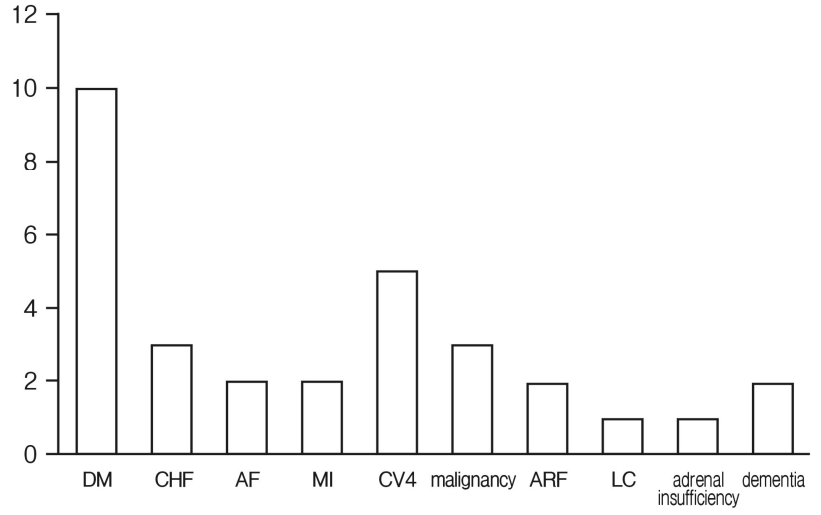

Fig. 1. Comorbidities of patients. DM: diabetes mellitus; CHF: congestive heart failure; AF: atrial fibrillation; MI: myocardial infarction; ARF: acute renal failure; LC: liver cirrhosis.

\section{Probable Origin of SEA}

Five patients experienced the development of SEA during the course of antibiotic treatment for other infections (e.g., sepsis, cerebellar abscess, pneumonia, pulmonary abscess, and pulmonary tuberculosis). Another 4 patients had a history of previous spinal intervention, such as epidural block, which was suspected as a cause of direct inoculation. The time interval from epidural intervention to admission was an average of 38.5 days (14-90 days). Three patients had a history of spinal fusion surgery (10 days- 4 months ago), and their SEA likely resulted from postoperative infection. Detailed predisposing conditions and final neurologic status are summarized in Table 4. The systemic infection group showed significantly worse outcomes than the patients who had epidural block or spinal surgery $(\mathrm{p}=0.010)$.

\section{Surgeny}

Open surgery was performed in 14 of 16 patients. The time to surgery ranged from 0 to 40 days. Two patients underwent delayed surgery in the middle of medical treatment due to neurological deterioration. Another 2 patients had delayed surgical intervention due to poor general condition caused by other systemic infections. Laminectomy and abscess removal were performed for 8 patients. Three patients underwent combined surgery via anterior and posterior approaches due to the presence of an extremely large abscess accompanied by bony destruction. Foreign body (pedicle screws) removal was performed for 3 patients who had undergone spinal fusion surgery (Table 1).

\section{Causative Organism}

Methicillin-resistant Staphylococcus epidermidis (MRSE) was the most frequent organism isolated in our study, which was identified from abscess and blood cultures in 5 and 1 patients, respectively. The mycobacterium tuberculosis (MTB) and methicillin-resistant Staphylococcus aureus (MRSA) were identified in 4 and 3 patients, respectively. Methicillin-sensitive Staphylococcus aureus (MSSA) was identified in 2 patients. Ultimately, causative organisms were microbiologically identified in 14 of 16 patients. In the open surgery group, 12 of 14 patients showed a positive culture result from the intraoperatively acquired specimen. However, the culture result was negative in 2 patients who had an aspiration biopsy. Whereas a single causative organism was identified in 9 patients, 3 patients had SEA with more than one organism: MRSE and tuberculosis in 2 patients and MRSA and MSSA in 1 patient. The 1 patient who had MRSE and MTB also had an additional organism (Staphylococcus hominis) identified in their preoperative blood culture. The blood cultures also identified the causative organism in 7 of 13 patients. Different organisms were identified between abscess and blood culture in 2 patients (Table 5).

\section{Clinical Outcomes}

Seven patients completely improved and were able to look after their own affairs without assistance. Two patients were moderately disabled. Another 2 patients were severely disabled and 5 patients died during the course of antibiotic treatment.

One of the 5 patients died from aortic pseudoaneurysm due to relapsed paraspinal abscess, which was thought to represent a complication of SEA. Four other patients died due to comorbidities, which were less related to SEA. Two had heart 
Table 5. Causative organisms of patients

\begin{tabular}{|c|c|c|c|}
\hline \multirow{2}{*}{ No } & \multirow{2}{*}{ Predisposing conditions } & \multicolumn{2}{|c|}{ Identified organism } \\
\hline & & Abscess & Blood \\
\hline 1 & Epidural block & Streptococcus bovis & Staphylococcus capitis \\
\hline 2 & Sepsis & MSSA, MRSA & MSSA \\
\hline 3 & Cerebellar abscess & No growth & No growth \\
\hline 4 & Epidural block & MRSE, MTB & Staphylococcus hominis \\
\hline 5 & & MRSE, MTB & No growth \\
\hline 6 & & MTB & Not performed \\
\hline 7 & Epidural block & No growth & MSSE \\
\hline 8 & & No growth & No growth \\
\hline 9 & Pneumonia & MRSA & No growth \\
\hline 10 & & MSSA & MSSA \\
\hline 11 & Epidural block & MRSE & Not performed \\
\hline 12 & Spinal fusion & MRSA & Not performed \\
\hline 13 & Spinal fusion & MRSE & No growth \\
\hline 14 & Pulmonary abscess & No growth & Escherichia coli \\
\hline 15 & Spinal fusion & MRSE & MRSE \\
\hline 16 & Pulmonary MTB & MTB & No growth \\
\hline
\end{tabular}

MTB: mycobacterium tuberculosis; MSSA: methicillin-sensitive Staphylococcus aureus; MRSA: methicillin-resistant Staphylococcus aureus; MRSE: methicillin-resistant Staphylococcus epidermidis; MSSE: methicillin-sensitive Staphylococcus epidermidis.

Table 6. Time to operation vs. final neurologic status

\begin{tabular}{ccccc}
\hline \multirow{2}{*}{ Time to operation (day) } & \multicolumn{3}{c}{ Final neurological status } & \multirow{2}{*}{ Total number of patients $^{*}$} \\
\cline { 2 - 4 } & $0-2$ & $3-4$ & $5-6$ & 11 \\
$0-7$ & 7 & $2 m$ & 2 & 5 \\
28 & & 5 & 5 \\
\hline
\end{tabular}

The statistical significance was defined as $p=0.014$ (Fisher's exact test).

*Final neurological status 1 to 5 is from modified Rankin grade and 6 is death.

problems and died suddenly from acute myocardial infarctions. Another 2 patients died from acute respiratory distress syndrome and atrial fibrillation induced cerebral infarction, respectively. The relationships between neurological outcome and the comorbidity $(\mathrm{p}=0.026)$, predisposing conditions $(\mathrm{p}=0.010)$ and time to surgery $(\mathrm{p}=0.014)$ were statistically significant (Tables 3, 4, 6), but it was not significantly related to Bremer's grading system (Table 2 ).

\section{DISCUSSION}

\section{Clinical Manifestations}

The classic diagnostic triad of spinal pain, fever, and neurological deficits, defined as the initial description of SEA, is still considered the hallmark of this disease ${ }^{16,17)}$. However, only $25 \%$ of our patients presented with this classic triad, whereas $62.5 \%$ and $31.3 \%$ of patients exhibited back pain and fever, respectively. Accurate and timely diagnosis is paramount to the successful management and eventual resolution of SEA ${ }^{12,14)}$. A high index of suspicion remains necessary to diagnose SEA accurately and expeditiously ${ }^{5,17)}$. However, delays in or missed initial diagnoses, which contribute to poor outcomes, are reported in $11 \%$ to $75 \%$ of cases ${ }^{5,12,25,26)}$.

We also support that a high index of suspicion is essential for patients with such symptoms because the triad is usually a nonspecific finding ${ }^{28)}$. Davis et al. $^{16)}$ also described the nonspecific nature of early symptoms and its challenge to the early diagnosis of SEA. Thus, we believe that the role of MRI and laboratory tests should be emphasized, especially in elderly patients, because the clinical signs or subjective symptoms of SEA can be vague in elderly patients whose immune response is blunted ${ }^{19)}$. Moreover, the clinical status can deteriorate irreversibly unrecognized and its progression can be faster than expected in elderly patients. MRI, especially in combination with gadolinium, represents the method of first choice for identification of SEA, making other diagnostic procedures essentially superfluous $^{26)}$.

In our study, female patients more frequently presented with 
SEA (10:6) compared to previous reports ${ }^{1,26)}$. All large case series of SEA revealed a preference for males in the development of SEA ${ }^{26)}$. Reihsaus et al. ${ }^{26)}$ reported a male preponderance in their meta-analysis of 915 cases, describing the male : female ratio as 1:0.56. They assumed that different risk factors, including alcohol abuse, use of IV drugs, and trauma, are partially responsible $^{26)}$. Adogwa et al. ${ }^{1)}$ also demonstrated a male preponderance in SEA patients 50 years of age and older, reporting a male:female ratio of 1:0.78.

However, the composition of the population in Korea is likely to be different from other countries, where the aforementioned studies were conducted. Because of the longer life expectancy of women in Korea, the proportion of women in the elderly population is different from the general population. We believe that this increased proportion of females in the elderly group results in the sex reversal observed in our study. Cho et $\mathrm{al}^{11)}$ reported that the life-time prevalence of low back pain was higher in female and was significantly related to advanced age and female sex in a study of a Korean rural com munity. Thus, it may be assumed that elderly females more frequently suffer from back pain and undergo invasive spinal intervention, which increases the risk of secondary or procedure-related SEA. Our study showed 7 of 16 patients had SEA that was related to invasive procedures.

\section{Treatment}

Although a combination of surgical drainage and antibiotics has been the treatment of choice ${ }^{3,8,12-14}$, there are no established guidelines about medical and/or surgical treatment for SEA. Some recommend that medical management be considered in patients with no neurological deficits, complete deficit for more than $72 \mathrm{hr}$, extensive multilevel abscess formation or in patients with severe concomitant diseases ${ }^{5}$. However, those on medical treatment should be carefully monitored on a regular basis because they may develop rapid and irreversible neurological deterioration ${ }^{19)}$. In our study, 2 patient (12.5\%) underwent delayed surgery in the middle of medical treatment due to neurological deterioration. Medical management was found to have a failure rate ranging from $6 \%$ to $49 \%$, possibly due to differences in patient selection ${ }^{5,6)}$. Patel et al. ${ }^{25)}$ found that diabetes mellitus (DM), CRP levels, white blood cell count, and bacteremia were predictors of failure in medical management and reported that $8.3 \%$ to $76.9 \%$ of failure rate was due to these risk factors.

Our results revealed that delayed surgery was related to poor outcomes among elderly SEA patients. However, the usefulness of early surgery in SEA is still debatable. Alton et al. ${ }^{4}$ reported that early surgery resulted in improved posttreatment neurologic outcomes, and the failure rate of medical management was high (75\%). Patel et al. ${ }^{25)}$ also reported that early surgery improved neurologic outcome compared to surgical treatment delayed by trial of medical management. Curry et $\mathrm{al}^{13)}$ and Hlavin et al. ${ }^{20)}$ also recommended early surgical inter- vention. In contrast, Karikari et al. ${ }^{22)}$ reported that early surgery was not associated with better outcome. Adogwa et al. ${ }^{1)}$ also found that early surgical decompression was not associated with superior clinical outcome, compared to antibiotic therapy alone in patients 50 years of age and order.

We believe that these discrepancies primarily stem from the selection bias of the patients. SEA comprises a wide range of conditions, including size, location, causative pathogen, and neurological status. We think that such heterogeneity of included study patients makes it difficult to identify widely acceptable guidelines. Thus, we are cautious about applying the information derived from other studies directly to our elderly patient group because their predisposing conditions, including age, comorbidities, and neurologic status, are different from those of other study populations. Therefore, we believe the treatment should be individualized and should consider each patient's conditions.

\section{Causative Organism}

Staphylococcus is the principle etiologic agent of SEA, and its proportion ranges from $59.2 \%$ to $93 \%$ in large case series ${ }^{6,12,13,26)}$. Adogawa et al. ${ }^{1}$ reported MSSA, MRSA, and coagulase-negative staphylococcus in $28 \%, 28 \%$, and $11 \%$ of cases, respectively. Reihsaus et al. ${ }^{26)}$ described the identification of Staphylococcal aureus (S. aureus) and Staphylococcus epidermidis (S. epidermidis) in 551 and 9 patients, respectively, in their meta-analysis of 915 patients. Del Curling et al. ${ }^{17)}$ and Hlavin et al. ${ }^{20)}$ also reported the ratio of $\mathrm{S}$. aureus: $\mathrm{S}$. epidermidis as 13:1, and 12:1, respectively. Whereas $S$. aureus is much more prevalent than $S$. epidermidis ${ }^{17,20,26)}$, they showed the same incidence as our results. This relatively high incidence of MRSE might have originated from the large proportion of procedurerelated SEA in our series, although the causality is not clear. Four of 5 MRSE patients had a previous history of epidural block or spinal fusion surgery.

Three patients presented with concomitant infection with multiple organisms. However, preoperative blood culture failed to isolate all organisms, and their superinfection was identified from the culture of the abscess acquired intraoperatively. We believe that the positive blood culture does not exclude the presence of other concomitant organisms in SEA. Thus, we recommend holding the initiation of antibiotics until a surgical specimen is acquired. Although we do not completely understand the mechanism of these superinfections, decreased general conditions in elderly patients may be one reason for it.

The accuracy of closed vertebral needle biopsy in patients with spondylitis without neurological deficit varies and has been reported to be approximately $70 \%{ }^{19)}$. Although we performed aspiration biopsy in 2 patients, none had microbiological confirmation of pathogens from the aspirated abscess. We think that previous antibiotic treatment might be the cause of a negative result, although we stopped the antibiotics several days before the biopsy. Negative results may be due to 
insufficient tissue or to the administration of antibiotics prior to biopsy ${ }^{19)}$.

Two patients in our study exhibited concomitant infection with MRSE and tuberculosis, which is very rare and has only been reported in a few cases ${ }^{23,24)}$. Tuberculous spondylitis is more prevalent in females and risk factors include $\mathrm{DM}^{23)}$. In our study, 2 patients were female, and one had DM. The rarity results in limited awareness of this concomitant infection, and the time required to obtain culture results varies by pathogens ${ }^{23)}$. For this reason, diagnosis is difficult and can sometimes be delayed $^{23}$. However, a mycobacterial culture and histopathological examination are recommended for all suspicious cases, even when pyogenic bacteria have already been isolated ${ }^{24)}$. Moreover, high suspicion is indispensable for those who show a poor response to adequate antibiotics ${ }^{23)}$.

\section{Clinical Outcome}

Neurological deficits caused by SEA are reported as a predictor of clinical outcome in some literature ${ }^{25,27)}$. However, this variable was not statistically significant in our study (Table 2). Adogwa et al. ${ }^{1)}$ also reported that an increasing baseline level of pain, neurologic deficits, and dorsally located SEA were independently associated with poor outcome in patients 50 years of age and older. Thirteen patients exhibited ventral SEA, and 2 patients had dorsal SEA in our cohort. Karikari et al. ${ }^{22)}$ also described that $30.6 \%$ of patients with dorsal SEA were paraplegic or quadriplegic, while only $7.3 \%$ of the patients with ventral SEA were paraplegic or quadriplegic $(p=0.003)$.

Most of our patients (75\%) had one or more comorbidities, which were statistically significantly related to final outcomes (Table 3). Anand et al. ${ }^{5}$ ) found that greater than 50\% of SEA patients had risk factors, such as DM, cancer, degenerative spinal disease, tumor, IV drug abuse, alcoholism, and steroid intake. Ten and 3 of our patients had DM and cancer history, respectively (Fig. 1). Chen et al. ${ }^{10)}$ found that underlying comorbid illness estimated by Charlson score was predictive of a poor prognosis with treatment failure or mortality. However, it is unclear whether the comorbidities are the cause of poor general conditions or vice versa in elderly patients.

The mortality rate in our study was relatively high at 31\% compared to other studies ${ }^{1,3,14,17,20)}$. We believe that our high mortality is primarily because of the older age and comorbidities of this cohort. Akalan and Ozgen ${ }^{3)}$ reported a 5.8\% mortality rate in their 36 SEA patients. However, direct comparison to our patients would be difficult because their mean age was 24.3 years old and only $16.6 \%$ had comorbidities ${ }^{3)}$. Hlavin et $\mathrm{al}^{20)}$ documented $23 \%$ mortality in 39 patients with SEA. More than half of these patients were less than 60 years old, although $87.1 \%$ did have comorbidities ${ }^{20)}$. Adogwa et al. ${ }^{1)}$ also reported a $25.6 \%$ mortality rate in patients 50 years of age and older. Whereas sepsis was the cause of $70 \%$ of the mortality in their study, 30\% was unrelated to SEA (e.g., end-stage renal disease, pulmonary embolism, and myocardial infarction) ${ }^{1)}$.
Most of our mortality was also not directly related to SEA, with $80 \%$ of our mortality being due to myocardial infarction, cerebral infarction, and pulmonary pathology.

\section{Limitations of the Study}

Our study has limitations that stem from the small number of included patients. For this reason, some important findings might have been statistically insignificant, and the power of conclusion may be weak, although some parameters including comorbidity, predisposing conditions and time to surgery were statistically significant. Moreover, our study population included a heterogeneous group with secondary or procedurerelated SEA and concomitant infection with tuberculosis. This heterogeneity likely diluted and masked some significant effects. Second, the retrospective study design may have caused selection bias, inaccuracy of data and neglect or missing of useful information. Although various radiological or laboratory tests were performed, we also acquired information, including comorbidities or past medical history, by interview or medical record review. There also might have been underreporting of patientreported information or mistakes in the communication process.

Although elderly patients have historically been a minor subgroup in the overall general population, their number is currently increasing, and new problems are emerging with increased longevity of the general population. SEA in the elderly population requires different management strategies due to their comorbidities. Therefore, we believe that additional large scale studies are indispensable for this population.

\section{CONCLUSION}

SEA remains a potentially fatal condition in elderly patients. The consideration of comorbidities is critical because they are an important cause of mortality. We identified a statistically significant relationship between neurological outcome and comorbidity $(\mathrm{p}=0.026)$, predisposing conditions $(\mathrm{p}=0.010)$ and time to surgery $(\mathrm{p}=0.014)$, suggesting that early surgery should be considered, even in elderly SEA patients.

\section{CONFLICTS OF INTEREST}

No potential conflict of interest relevant to this article was reported.

\section{REFERENCES}

1. Adogwa O, Karikari IO, Carr KR, Krucoff M, Ajay D, Fatemi $\mathrm{P}$, et al.: Spontaneous spinal epidural abscess in patients 50 years of age and older: A 15-year institutional perspective and review of the literature: Clinical article. J Neurosurg Spine 20:344-349, 2014 
2. Ahl T, Hedström M, von Heijne A, Hammers Stiernstedt S: Acute spinal epidural abscess without concurrent spondylodiscitis. Successful closed treatment in 10 cases. Acta Orthop Scand 70:199-202, 1999

3. Akalan N, Ozgen T: Infection as a cause of spinal cord compression: A review of 36 spinal epidural abscess cases. Acta Neurochir (Wien) 142:17-23, 2000

4. Alton TB, Patel AR, Bransford RJ, Bellabarba C, Lee MJ, Chapman JR: Is there a difference in neurologic outcome in medical versus early operative management of cervical epidural abscesses? Spine J 15:10-17, 2015

5. Anand S, Maini L, Agarwal A, Singh T, Dhal AK, Dhaon BK: Spinal epidural abscess. A report of six cases. Int Orthop 23:175177, 1999

6. Arko Lt, Quach E, Nguyen V, Chang D, Sukul V, Kim BS: Medical and surgical management of spinal epidural abscess: A systematic review. Neurosurg Focus 37:E4, 2014

7. Bremer AA, Darouiche RO: Spinal epidural abscess presenting as intra-abdominal pathology: A case report and literature review. J Emerg Med 26:51-56, 2004

8. Chang WC, Tsou HK, Kao TH, Yang MY, Shen CC: Successful treatment of extended epidural abscess and long segment osteomyelitis: A case report and review of the literature. Surg Neurol 69:117-120, 2008

9. Charlson ME, Pompei P, Ales KI, MacKenzie CR: A new method of classifying prognostic comorbidity in longitudinal studies: Development and validation. J Chronic Dis 40:373-383, 1987

10. Chen WC, Wang JL, Wang JT, Chen YC, Chang SC: Spinal epidural abscess due to Staphylococcus aureus: Clinical manifestations and outcomes. J Microbiol Immunol Infect 41:215-221, 2008

11. Cho NH, Jung YO, Lim SH, Chung CK, Kim HA: The prevalence and risk factors of low back pain in rural community residents of Korea. Spine (Phila Pa 1976) 37:2001-2010, 2012

12. Connor DE, Jr., Chittiboina P, Caldito G, Nanda A: Comparison of operative and nonoperative management of spinal epidural abscess: A retrospective review of clinical and laboratory predictors of neurological outcome. J Neurosurg Spine 19:119127, 2013

13. Curry WT, Jr., Hoh BL, Amin-Hanjani S, Eskandar EN: Spinal epidural abscess: Clinical presentation, management, and outcome. Surg Neurol 63:364-371, 2005

14. Danner RL, Hartman BJ: Update on spinal epidural abscess: 35 cases and review of the literature. Rev Infect Dis 9:265-274, 1987

15. Darouiche RO: Spinal epidural abscess. N Engl J Med 355:
2012-2020, 2006

16. Davis DP, Wold RM, Patel RJ, Tran AJ, Tokhi RN, Chan TC, et al.: The clinical presentation and impact of diagnostic delays on emergency department patients with spinal epidural abscess. J Emerg Med 26:285-291, 2004

17. Del Curling O, Jr., Gower DJ, McWhorter JM: Changing concepts in spinal epidural abscess: A report of 29 cases. Neurosurgery 27:185-192, 1990

18. Ericsson M, Algers G, Schliamser SE: Spinal epidural abscesses in adults: Review and report of iatrogenic cases. Scand J Infect Dis 22:249-257, 1990

19. Govender S: Spinal infections. J Bone Joint Surg Br 87:14541458, 2005

20. Hlavin ML, Kaminski HJ, Ross JS, Ganz E: Spinal epidural abscess: A ten-year perspective. Neurosurgery 27:177-184, 1990

21. Huang YY, Zuo Z, Yuan HB, Tsou MY, Chen MT, Tsai SK: A paraspinal abscess following spinal anaesthesia for caesarean section and patient-controlled epidural analgesia for postoperative pain. Int J Obstet Anesth 14:252-255, 2005

22. Karikari IO, Powers CJ, Reynolds RM, Mehta AI, Isaacs RE: Management of a spontaneous spinal epidural abscess: A singlecenter 10-year experience. Neurosurgery 65:919-923, 2009

23. Kim YM, Cha JH: Delayed diagnosis of tuberculous spondylitis masked by concomitant methicillin resistant Staphylococcus aureus infection. J Korean Neurosurg Soc 47:235-238, 2010

24. Mousa HA: Concomitant spine infection with mycobacterium tuberculosis and pyogenic bacteria: A case report. Spine (Phila Pa 1976) 28:E152-E154, 2003

25. Patel AR, Alton TB, Bransford RJ, Lee MJ, Bellabarba CB, Chapman JR: Spinal epidural abscesses: Risk factors, medical versus surgical management, a retrospective review of 128 cases. Spine J 14:326-330, 2014

26. Reihsaus E, Waldbaur H, Seeling W: Spinal epidural abscess: A meta-analysis of 915 patients. Neurosurg Rev 23:175-204, 2000

27. Roßbach BP, Niethammer TR, Paulus AC, Melcher C, Birkenmaier C, Jansson V, et al.: Surgical treatment of patients with spondylodiscitis and neurological deficits caused by spinal epidural abscess (SEA) is a predictor of clinical outcome. J Spinal Disord Tech 27:395-400, 2014

28. Shweikeh F, Saeed K, Bukavina L, Zyck S, Drazin D, Steinmetz MP: An institutional series and contemporary review of bacterial spinal epidural abscess: Current status and future directions. Neurosurg Focus 37:E9, 2014 